TRANSACTIONS OF THE

AMERICAN MATHEMATICAL SOCIETY

Volume 359, Number 5, May 2007, Pages 2043-2058

S 0002-9947(06)03987-0

Article electronically published on December 19, 2006

\title{
A MODEL CATEGORY STRUCTURE ON THE CATEGORY OF SIMPLICIAL CATEGORIES
}

\author{
JULIA E. BERGNER
}

\begin{abstract}
In this paper we put a cofibrantly generated model category structure on the category of small simplicial categories. The weak equivalences are a simplicial analogue of the notion of equivalence of categories.
\end{abstract}

\section{INTRODUCTION}

Simplicial categories, which in this paper we will take to mean categories enriched over simplicial sets, arise in the study of homotopy theories. Given any model category $\mathcal{M}$, the simplicial localization of $\mathcal{M}$ as given in 4 is a simplicial category which possesses the homotopy-theoretic information contained in $\mathcal{M}$. Finding a model category structure on the category of simplicial categories is then the first step in studying the homotopy theory of homotopy theories.

In an early online version of the book [2, Dwyer, Hirschhorn, and Kan present a cofibrantly generated model category structure on the category of simplicial categories, but as Toën and Vezzosi point out in their paper [10, this model category structure is incorrect, in that some of the proposed generating acyclic cofibrations are not actually weak equivalences. Here we complete the work of [2] by describing a different set of generating acyclic cofibrations which are in fact weak equivalences and which, along with the generating cofibrations given in 2, enable us to prove that the desired model category structure exists.

There are several contexts in which having such a model category structure is helpful. In [1, we show that this model structure is Quillen equivalent to three other model category structures. These Quillen equivalences are of interest in homotopy theory as well as higher category theory. Toën and Vezzosi also use simplicial categories in their work on homotopical algebraic geometry [10].

Note that the term "simplicial category" is potentially confusing. As we have already stated, by a simplicial category we mean a category enriched over simplicial sets. If $a$ and $b$ are objects in a simplicial category $\mathcal{C}$, then we denote by $\operatorname{Hom}_{\mathcal{C}}(a, b)$ the function complex, or simplicial set of maps $a \rightarrow b$ in $\mathcal{C}$. This notion is more restrictive than that of a simplicial object in the category of categories. Using our definition, a simplicial category is essentially a simplicial object in the category of categories which satisfies the additional condition that all the simplicial operators induce the identity map on the objects of the categories involved [3, 2.1].

We will assume that our simplicial categories are small, namely, that they have a set of objects. A functor between two simplicial categories $f: \mathcal{C} \rightarrow \mathcal{D}$ consists of

Received by the editors January 28, 2005.

2000 Mathematics Subject Classification. Primary 18G55, 18D20.

(c)2006 American Mathematical Society Reverts to public domain 28 years from publication 
a map of sets $f: O b(\mathcal{C}) \rightarrow O b(\mathcal{D})$ on the objects of the two simplicial categories, and function complex maps $f: \operatorname{Hom}_{\mathcal{C}}(a, b) \rightarrow \operatorname{Hom}_{\mathcal{D}}(f a, f b)$ which are compatible with composition. Let $\mathcal{S} \mathcal{C}$ denote the category whose objects are the small simplicial categories and whose morphisms are the functors between them. This category SC is the underlying category of our model category structure.

In a similar way, we can consider categories enriched over the category of topological spaces. Making slight modifications to the ideas from this paper, it is possible to put an analogous model category structure on the category of small topological categories.

Recall that a model category structure on a category $\mathcal{C}$ is a choice of three distinguished classes of morphisms, namely, fibrations, cofibrations, and weak equivalences. We use the term acyclic (co)fibration to denote a map which is both a (co)fibration and a weak equivalence. This structure is required to satisfy five axioms [5, 3.3].

Before defining these three classes of morphisms in $\mathcal{S} \mathcal{C}$, we need some notation. Suppose that $\mathcal{C}$ and $\mathcal{D}$ are two simplicial categories. Let $\pi_{0} \mathcal{C}$ denote the category of components of $\mathcal{C}$, namely, the category in which the objects are the same as those of $\mathcal{C}$ and the morphisms between objects $a$ and $b$ are given by

$$
\operatorname{Hom}_{\pi_{0}} \mathrm{e}(a, b)=\pi_{0} \operatorname{Hom}_{\mathfrak{C}}(a, b) .
$$

If $f: \mathcal{C} \rightarrow \mathcal{D}$ is a map of simplicial categories, then $\pi_{0} f: \pi_{0} \mathcal{C} \rightarrow \pi_{0} \mathcal{D}$ denotes the induced map on the categories of components of $\mathcal{C}$ and $\mathcal{D}$.

If $\mathcal{C}$ is a simplicial category, say that a morphism $e \in \operatorname{Hom}_{\mathcal{C}}(a, b)_{0}$ is a homotopy equivalence if it becomes an isomorphism in $\pi_{0} \mathrm{C}$.

Now, given these definitions, our three classes of morphisms are defined as follows.

(1) The weak equivalences are the maps $f: \mathcal{C} \rightarrow \mathcal{D}$ satisfying the following two conditions:

- (W1) For any objects $a_{1}$ and $a_{2}$ in $\mathcal{C}$, the map

$$
\operatorname{Hom}_{\mathcal{C}}\left(a_{1}, a_{2}\right) \rightarrow \operatorname{Hom}_{\mathcal{D}}\left(f a_{1}, f a_{2}\right)
$$

is a weak equivalence of simplicial sets.

- (W2) The induced functor $\pi_{0} f: \pi_{0} \mathcal{C} \rightarrow \pi_{0} \mathcal{D}$ is an equivalence of categories.

(2) The fibrations are the maps $f: \mathcal{C} \rightarrow \mathcal{D}$ satisfying the following two conditions:

- (F1) For any objects $a_{1}$ and $a_{2}$ in $\mathcal{C}$, the map

$$
\operatorname{Hom}_{\mathcal{C}}\left(a_{1}, a_{2}\right) \rightarrow \operatorname{Hom}_{\mathcal{D}}\left(f a_{1}, f a_{2}\right)
$$

is a fibration of simplicial sets.

- (F2) For any object $a_{1}$ in $\mathcal{C}, b$ in $\mathcal{D}$, and homotopy equivalence $e$ : $f a_{1} \rightarrow b$ in $\mathcal{D}$, there is an object $a_{2}$ in $\mathcal{C}$ and homotopy equivalence $d: a_{1} \rightarrow a_{2}$ in $\mathcal{C}$ such that $f d=e$.

(3) The cofibrations are the maps which have the left lifting property with respect to the maps which are both fibrations and weak equivalences.

The weak equivalences are often called DK-equivalences, as they were first described by Dwyer and Kan in [3]. They are a generalization of the notion of equivalence of categories to the simplicial setting.

We now state our main theorem. 
Theorem 1.1. There is a model category structure on the category Se of all small simplicial categories with the above weak equivalences, fibrations, and cofibrations.

We will actually prove the stronger statement that the above model category structure is cofibrantly generated. Recall that a cofibrantly generated model category is one for which there are two specified sets of morphisms, one of generating cofibrations and one of generating acyclic cofibrations, such that a map is a fibration if and only if it has the right lifting property with respect to the generating acyclic cofibrations, and a map is an acyclic fibration if and only if it has the right lifting property with respect to the generating cofibrations. For more details about cofibrantly generated model category structures, see [7, Ch. 11]. To prove the theorem, we will use the following proposition, which is stated in a more general form by Hirschhorn [7, 11.3.1].

Proposition 1.2. Let $\mathcal{M}$ be a category with all small limits and colimits and specified classes of weak equivalences and fibrations. Define a map to be a cofibration if it has the left lifting property with respect to the acyclic fibrations. Suppose that the class of weak equivalences is closed under retracts and satisfies the "two out of three property" [5. 3.3]. Suppose further that there exist sets $C$ and $A$ of maps in $\mathcal{M}$ satisfying the following properties:

(1) Both $C$ and $A$ permit the small object argument [7, 10.5.15].

(2) A map is a fibration if and only if it has the right lifting property with respect to the maps in $A$.

(3) A map is an acyclic fibration if and only if it has the right lifting property with respect to the maps in $C$.

(4) A map is an acyclic cofibration if and only if it has the left lifting property with respect to the fibrations.

Then there is a cofibrantly generated model category structure on $\mathcal{M}$ in which $C$ is a set of generating cofibrations and $A$ is a set of generating acyclic cofibrations.

Let SSets denote the category of simplicial sets. Recall in SSets we have for any $n \geq 0$ the $n$-simplex $\Delta[n]$, its boundary $\dot{\Delta}[n]$, and, for any $0 \leq k \leq n, V[n, k]$, which is $\dot{\Delta}[n]$ with the $k$ th face removed. Given a simplicial set $X$, we denote by $|X|$ its geometric realization. The standard model category structure on SSets is cofibrantly generated; the generating cofibrations are the maps $\dot{\Delta}[n] \rightarrow \Delta[n]$ for $n \geq 0$, and the generating acyclic cofibrations are the maps $V[n, k] \rightarrow \Delta[n]$ for $n \geq 1$ and $0 \leq k \leq n$. More details on simplicial sets and the model category structure on them can be found in [6].

There is a functor

$$
U: \text { SSets } \rightarrow \text { SC }
$$

which takes a simplicial set $X$ to the category with objects $x$ and $y$ and with $\operatorname{Hom}(x, y)=X$ but no other nonidentity morphisms.

We will say that a simplicial set $K$ is weakly contractible if all the homotopy groups of $|K|$ are trivial.

We will refer to the model category structure on the category of simplicial categories with a fixed set $\mathcal{O}$ of objects, denoted $\mathcal{S C}_{\mathcal{O}}$, such that all the morphisms induce the identity map on the objects, as defined by Dwyer and Kan in [4]. The weak equivalences are the maps satisfying condition W1 and the fibrations are the maps satisfying condition F1. 
We can then define our generating cofibrations and acyclic cofibrations as follows. The generating cofibrations are the maps:

- (C1) $U \dot{\Delta}[n] \rightarrow U \Delta[n]$ for $n \geq 0$, and

- (C2) $\phi \rightarrow\{x\}$, where $\phi$ is the simplicial category with no objects and $\{x\}$ denotes the simplicial category with one object $x$ and no nonidentity morphisms.

The generating acyclic cofibrations are:

- (A1) the maps $U V[n, k] \rightarrow U \Delta[n]$ for $n \geq 1$, and

- (A2) inclusion maps $\{x\} \rightarrow \mathcal{H}$ which are DK-equivalences, where $\{x\}$ is as in $\mathrm{C} 2$ and $\{\mathcal{H}\}$ is a set of representatives for the isomorphism classes of simplicial categories with two objects $x$ and $y$, weakly contractible function complexes, and only countably many simplices in each function complex. Furthermore, we require that the inclusion map $\{x\} \amalg\{y\} \rightarrow \mathcal{H}$ be a cofibration in $\mathcal{S C}_{\{x, y\}}$.

Notice that it follows from the definition of DK-equivalence that the map $\{x\} \rightarrow$ $\pi_{0} \mathcal{H}$ is an equivalence of categories. In particular, all 0 -simplices of the function complexes of $\mathcal{H}$ are homotopy equivalences.

The idea behind the set A2 of generating acyclic cofibrations is the fact that two simplicial categories can have a weak equivalence between them which is not a bijection on objects, much as two categories can be equivalent even if they do not have the same objects. We only require that our weak equivalences are surjective on equivalence classes of objects. Thus, we must consider acyclic cofibrations for which the object sets are not isomorphic. The requirement that there be only countably many simplices is included so that we have a set rather than a proper class of maps.

We also show that this model structure is right proper. Recall that a model category is right proper if every pullback of a weak equivalence along a fibration is a weak equivalence. It would be helpful to know if this model structure is also left proper, namely, that every pushout of a weak equivalence along a cofibration is a weak equivalence. We do not know if this condition holds for $\mathcal{S}$. It would also be useful to know that $\mathcal{S C}$ had the additional structure of a simplicial model category, but we currently do not know of such a structure.

In section 2, we show that these proposed generating acyclic cofibrations satisfy the necessary conditions to be a generating set. In section 3 , we complete the proof of Theorem 1.1 and show that the model structure is right proper. In section 4, we prove a technical lemma that we needed in section 2 .

\section{The Generating ACYCLIC COFIBRATions}

In this section, we will show that a map in Se satisfies properties F1 and F2 if and only if it has the right lifting property with respect to the maps in A1 and A2.

We begin by stating some facts about the Dwyer-Kan model category structure $\mathcal{S C}_{\mathcal{O}}$ on the category of simplicial categories with a fixed set $\mathcal{O}$ of objects. The weak equivalences are the maps which satisfy property $\mathrm{W} 1$, and the fibrations are the maps which satisfy property F1. The cofibrations are the maps which have the left lifting property with respect to the acyclic fibrations. However, we would like a more explicit description of the cofibrations in this category, for which we need some definitions. If $\mathcal{C}$ is a simplicial category, then let $\mathcal{C}_{k}$ denote the (discrete) category whose morphisms are the $k$-simplices of the morphisms of $\mathcal{C}$. 
Definition 2.1 (4 $4,7.4])$. A map $f: \mathcal{C} \rightarrow \mathcal{D}$ in $\mathcal{S C}_{\mathcal{O}}$ is free if

(1) $f$ is a monomorphism,

(2) if $*$ denotes the free product, then in each simplicial dimension $k$, the category $\mathcal{D}_{k}$ admits a unique free factorization $\mathcal{D}_{k}=f\left(\mathcal{C}_{k}\right) * \mathcal{F}_{k}$, where $\mathcal{F}_{k}$ is a free category, and

(3) for each $k \geq 0$, all degeneracies of generators of $\mathcal{F}_{k}$ are generators of $\mathcal{F}_{k+1}$.

Definition 2.2 ([4, 7.5]). A map $f: \mathcal{C} \rightarrow \mathcal{D}$ of simplicial categories is a strong retract of a map $f^{\prime}: \mathcal{C} \rightarrow \mathcal{D}^{\prime}$ if there exists a commutative diagram

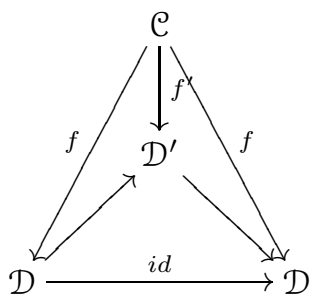

Then, we have $[4,7.6]$ that the cofibrations of $\mathcal{S C}_{\mathcal{O}}$ are precisely the strong retracts of free maps. In particular, a cofibrant simplicial category is a retract of a free category.

Given these facts, we now continue with our discussion of the generating acyclic cofibrations.

Recall (1) the map $U:$ SSets $\rightarrow$ SC. We first consider the set A1 of maps $U V[n, k] \rightarrow U \Delta[n]$ for $n \geq 1$ and $0 \leq k \leq n$. Using the model category structure on simplicial sets, we can see that a map of simplicial categories has the right lifting property with respect to the maps in A1 if and only if it satisfies the property F1.

We then consider the maps in A2 which we would also like to be generating acyclic cofibrations and show that maps with the right lifting property with respect to the maps in A1 and A2 are precisely the maps which satisfy conditions F1 and F2. The proof of this statement will take up the remainder of this section, and we will treat each implication separately.

Proposition 2.3. Suppose that a map $f: \mathcal{C} \rightarrow \mathcal{D}$ of simplicial categories has the right lifting property with respect to the maps in A1 and A2. Then $f$ satisfies condition F2.

Before proving this proposition, we state a lemma.

Lemma 2.4. Let $\mathcal{F}$ be a (discrete) simplicial category with object set $\{x, y\}$ and one nonidentity morphism $g: x \rightarrow y$. Let $\mathcal{E}^{\prime}$ be a simplicial category also with object set $\{x, y\}$. Let $i: \mathcal{F} \rightarrow \mathcal{E}^{\prime}$ send $g$ to a homotopy equivalence in $H_{o} m_{\mathcal{E}^{\prime}}(x, y)$. This map $i$ can be factored as a composite $\mathcal{F} \rightarrow \mathcal{H} \rightarrow \mathcal{E}^{\prime}$ in such a way that the composite map $\{x\} \rightarrow \mathcal{F} \rightarrow \mathcal{H}$ is isomorphic to a map in A2.

We will prove this lemma in section 4 . We now prove Proposition 2.3 assuming Lemma 2.4

Proof of Proposition 2.3. Given objects $a_{1}$ in $\mathcal{C}$ and $b$ in $\mathcal{D}$, we need to show that a homotopy equivalence $e: f a_{1} \rightarrow b$ in $\mathcal{D}$ lifts to a homotopy equivalence $d: a_{1} \rightarrow a_{2}$ for some $a_{2}$ in $\mathcal{C}$ such that $f a_{2}=b$ and $f d=e$. So, we begin by considering the objects $a=f a_{1}$ and $b$ in $\mathcal{D}$. 
We first consider the case where $a \neq b$. Define $\mathcal{E}^{\prime}$ to be the full simplicial subcategory of $\mathcal{D}$ with objects $a=f a_{1}$ and $b$, and let $\mathcal{F}$ be a simplicial category with objects $a$ and $b$ and a single nonidentity morphism $g: a \rightarrow b$. Let $i: \mathcal{F} \rightarrow \mathcal{E}^{\prime}$ send $g$ to a homotopy equivalence $e: a \rightarrow b$. By Lemma 2.4 we can factor this map as $\mathcal{F} \rightarrow \mathcal{H} \rightarrow \mathcal{E}^{\prime}$ in such a way that the composite $\{a\} \rightarrow \mathcal{F} \rightarrow \mathcal{H}$ is isomorphic to a map in $\mathrm{A} 2$.

It follows that the composite $\left\{a_{1}\right\} \rightarrow\{a\} \rightarrow \mathcal{H}$ is also isomorphic to a map in A2. Then consider the composite $\mathcal{H} \rightarrow \mathcal{E}^{\prime} \rightarrow \mathcal{D}$ where the map $\mathcal{E}^{\prime} \rightarrow \mathcal{D}$ is the inclusion map. These maps fit into a diagram

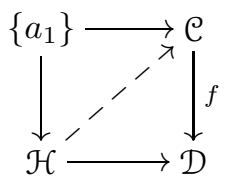

The lift exists because we assume that the map $f: \mathcal{C} \rightarrow \mathcal{D}$ has the right lifting property with respect to all maps in A2. Now, composing the map $\mathcal{F} \rightarrow \mathcal{H}$ with the lift sends the map $g$ in $\mathcal{F}$ to a map $d$ in $\mathcal{C}$ such that $f d=e$. The map $d$ is a homotopy equivalence since all the morphisms of $\mathcal{H}$ are homotopy equivalences and therefore map to homotopy equivalences in $\mathcal{C}$.

Now suppose that $a=b$. Define $\mathcal{E}^{\prime}$ to be the simplicial category with two objects $a$ and $a^{\prime}$ such that each function complex of $\mathcal{E}^{\prime}$ is the $\operatorname{simplicial}$ set $\operatorname{Hom}_{\mathcal{D}}(a, a)$ and compositions are defined as they are in $\mathcal{D}$. We then define the map $\mathcal{E}^{\prime} \rightarrow \mathcal{D}$ which sends both objects of $\mathcal{E}^{\prime}$ to $a$ in $\mathcal{D}$ and is the identity map on all the function complexes. Given this simplicial category $\mathcal{E}^{\prime}$, the argument proceeds as above.

We now prove the converse.

Proposition 2.5. Suppose $f: \mathcal{C} \rightarrow \mathcal{D}$ is a map of simplicial categories which satisfies properties F1 and F2. Then $f$ has the right lifting property with respect to the maps in A2.

Again, we state a lemma before proceeding with the proof of this proposition.

Lemma 2.6. Suppose that $A \rightarrow B$ is a cofibration, $C \rightarrow D$ is a fibration, and $B^{\prime} \rightarrow B$ is a weak equivalence in a model category $\mathcal{M}$. Then in the following commutative diagram:

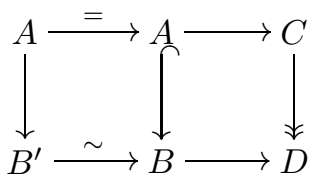

a lift $B \rightarrow C$ exists if and only if a lift $B^{\prime} \rightarrow C$ exists.

Proof. If the lift $B \rightarrow C$ exists, it follows that the lift $B^{\prime} \rightarrow C$ exists via composition with the map $B^{\prime} \rightarrow B$.

To prove the converse, we first note that the map $B^{\prime} \rightarrow B$ can be factored as the composite

$$
B^{\prime} \hookrightarrow B^{\prime \prime} \rightarrow B
$$

of a cofibration and a fibration, where each is a weak equivalence because the map $B^{\prime} \rightarrow B$ is. Thus the fact that $A \rightarrow B$ is a cofibration implies that there is a lift in 
the diagram

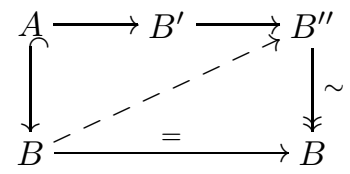

It now suffices to show that there is a lift in the diagram

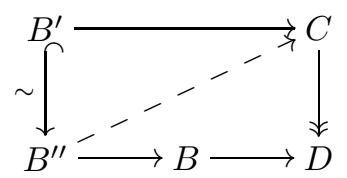

However, this fact again follows from the lifting properties of a model category.

We are now able to prove the proposition.

Proof of Proposition 2.5. We need to show that there exists a lift in any diagram of the form

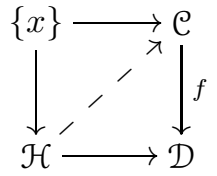

where $\{x\} \rightarrow \mathcal{H}$ is a map in A2. Given an object $a_{1}$ in $\mathcal{C}$ and a homotopy equivalence $e: a=f a_{1} \rightarrow b$, there exists an object $a_{2}$ in $\mathcal{C}$ and a homotopy equivalence $d: a_{1} \rightarrow a_{2}$ such that $f d=e$, since the map $f$ satisfies property F2.

Let $g: x \rightarrow y$ be a homotopy equivalence in $\mathcal{H}$. Let $\mathcal{F}$ denote the subcategory of $\mathcal{H}$ consisting of the objects $x$ and $y$ and $g$ its only nonidentity morphism. Consider the composite map $\{x\} \rightarrow \mathcal{F} \rightarrow \mathcal{H}$ and the resulting diagram

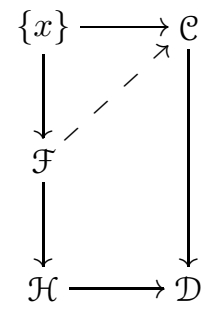

Because the map $\mathcal{F} \rightarrow \mathcal{D}$ factors through $\mathcal{H}$, which consists of homotopy equivalences, the image of $g$ in $\mathcal{D}$ is a homotopy equivalence. Thus, the existence of the lift in the above diagram follows from the fact that the map $f$ satisfies $\mathrm{F} 2$.

Now, we need to show that the rest of $\mathcal{H}$ lifts to $\mathcal{C}$. We begin by assuming that $a \neq b$ and therefore $a_{1} \neq a_{2}$. Consider the full simplicial subcategory of $\mathcal{C}$ with objects $a_{1}$ and $a_{2}$, and denote by $\mathrm{C}^{\prime}$ the isomorphic simplicial category with objects $x$ and $y$. Define $\mathcal{D}^{\prime}$ analogously where we take objects $x$ and $y$ rather than $a$ and $b$. Now, we can work in the category $\mathcal{S}_{\{x, y\}}$ of simplicial categories with fixed object set $\{x, y\}$. Note that the map $\mathcal{C}^{\prime} \rightarrow \mathcal{D}^{\prime}$ is still a fibration in $\mathcal{S} \mathcal{C}$, and in fact it is a 
fibration in $\mathcal{S}_{\{x, y\}}$. Now define $\mathcal{E}$ to be the pullback in the diagram

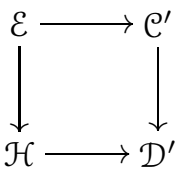

Then the map $\mathcal{E} \rightarrow \mathcal{H}$ is also a fibration in $\mathcal{S C}_{\{x, y\}}$ [5, 3.14(iii)].

By Lemma 2.4, we can factor the map $\mathcal{F} \rightarrow \mathcal{E}$ as the composite $\mathcal{F} \rightarrow \mathcal{H}^{\prime} \rightarrow \mathcal{E}$ for some simplicial category $\mathcal{H}^{\prime}$ such that the composite $\{x\} \rightarrow \mathcal{F} \rightarrow \mathcal{H}^{\prime}$ is isomorphic to a map in A2. Then, note that the composite map $\mathcal{H}^{\prime} \rightarrow \mathcal{E} \rightarrow \mathcal{H}$ is a weak equivalence in $\mathcal{S C}_{\{x, y\}}$ since all the function complexes of $\mathcal{H}$ and $\mathcal{H}^{\prime}$ are weakly contractible.

Now, we have a diagram

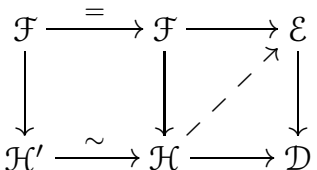

in which the dotted arrow lift exists by Lemma 2.6

If $a=b$, then $\mathcal{D}^{\prime}$ (and possibly $\mathrm{C}^{\prime}$ ) as defined above will have only one object $x$. If this is the case, then define the simplicial category $\mathcal{D}^{\prime \prime}$ with two objects $x$ and $y$ such that each function complex is the simplicial set $\operatorname{Hom}_{\mathcal{D}^{\prime}}(x, x)$ (as in the proof of Proposition 2.3). We can then factor the map $\mathcal{H} \rightarrow \mathcal{D}^{\prime}$ through the object $\mathcal{D}^{\prime \prime}$, where the map $\mathcal{D}^{\prime \prime} \rightarrow \mathcal{D}$ sends both objects of $\mathcal{D}^{\prime \prime}$ to $a$ in $\mathcal{D}$ and is the identity map on each function complex. If $\mathcal{C}^{\prime}$ also has one object, then we obtain a simplicial category $\mathcal{C}^{\prime \prime}$ in the same way. Then, we can repeat the argument above in the left-hand square of the diagram

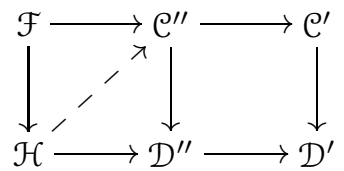

to obtain a lift $\mathcal{H} \rightarrow \mathrm{C}^{\prime \prime}$, and hence a lift $\mathcal{H} \rightarrow \mathrm{C}^{\prime}$ via composition.

\section{The MOdEL CATEgORY STRUCTURE}

In order to show that our proposed model category structure exists, we need to show that our definitions are compatible with one another. In particular, we need to prove that the maps with the left lifting property with respect to the fibrations are exactly the acyclic cofibrations, and that the maps with the right lifting property with respect to the generating cofibrations are exactly the maps which are fibrations and weak equivalences. Before proving these statements, however, we prove that first three model category axioms hold in SC.

Proposition 3.1. The category SC has all finite limits and colimits, and its class of weak equivalences is closed under retracts and satisfies the "two out of three" property.

Proof. It can be shown that the category of all simplicial categories has all coproducts and all coequalizers, and therefore all finite colimits, and all products and 
equalizers, and therefore all finite limits. To prove the existence of coequalizers, for example, we use the existence of coequalizers for sets (for the objects) and simplicial sets (for the morphisms). The two properties for the class of weak equivalences follow as usual, for example, as in [5, 8.10].

We first consider the sets $\mathrm{C} 1$ and $\mathrm{C} 2$. Suppose we have a map $f: \mathcal{C} \rightarrow \mathcal{D}$ which is a fibration and a weak equivalence. Using the model category of simplicial sets, we can see that a map satisfies conditions F1 and W1 if and only if it has the right lifting property with respect to the maps $U \dot{\Delta}[n] \rightarrow U \Delta[n]$ for $n \geq 0$, where $U$ is the map (11) from simplicial sets to simplicial categories defined in the first section.

However, the maps $U \dot{\Delta}[n] \rightarrow U \Delta[n]$ only generate those cofibrations between simplicial categories with the same number of objects, a condition that we do not require on our cofibrations of simplicial categories. Therefore, we include as a generating cofibration the map $\phi \rightarrow\{x\}$ from the simplicial category with no objects to the single-object simplicial category with no nonidentity morphisms. In other words, we are including the addition of an object as a cofibration.

Proposition 3.2. A map in Se is a fibration and a weak equivalence if and only if it has the right lifting property with respect to the maps in C1 and C2.

Proof. First suppose that $f: \mathcal{C} \rightarrow \mathcal{D}$ is both a fibration and a weak equivalence. By conditions F1 and W1, the map $\operatorname{Hom}_{\mathcal{C}}(a, b) \rightarrow \operatorname{Hom}_{\mathcal{D}}(f a, f b)$ is an acyclic fibration of simplicial sets for any choice of objects $a$ and $b$ in $\mathcal{C}$. In other words, there is a lift in any diagram of the form

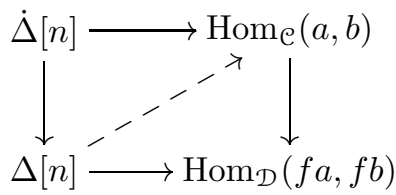

However, having this lift is equivalent to having a lift in the diagram

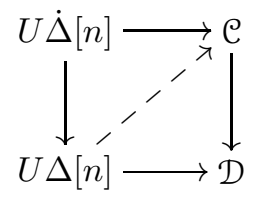

where the objects $x$ and $y$ of $U \dot{\Delta}[n]$ map to $a$ and $b$ in $\mathcal{C}$, and analogously for $U \Delta[n]$ and $\mathcal{D}$. Hence, $f$ has the right lifting property with respect to the maps in C1.

It remains only to show that $f$ has the right lifting property with respect to the map $\phi \rightarrow\{x\}$. However, this property is equivalent to $f$ being onto on objects. Being onto on homotopy equivalence classes of objects follows from condition W2. Then suppose that $e: a \rightarrow b$ is an isomorphism in $\mathcal{D}$ and there is an object $a_{1}$ in $\mathcal{C}$ such that $f a_{1}=a$. Since $e$ is a homotopy equivalence, by $\mathrm{F} 2$ there is a homotopy equivalence in $\mathcal{C}$ with domain $a_{1}$ and which maps to $e$ under $f$. In particular, there is an object $a_{2}$ in $\mathcal{C}$ mapping to $b$.

Conversely, suppose that $f$ has the right lifting property with respect to the maps in $\mathrm{C} 1$ and $\mathrm{C} 2$. Again, using the model category structure on simplicial sets, we have that the map

$$
\operatorname{Hom}_{\mathcal{C}}(a, b) \rightarrow \operatorname{Hom}_{\mathcal{D}}(f a, f b)
$$


is both a fibration and a weak equivalence, satisfying both F1 and W1. It follows that $\operatorname{Hom}_{\pi_{0}} \mathrm{e}(a, b) \rightarrow \operatorname{Hom}_{\pi_{0} \mathcal{D}}(f a, f b)$ is an isomorphism. As above, having the right lifting property with respect to the map $\phi \rightarrow\{x\}$ is equivalent to being onto on objects. These two facts show then that $\pi_{0} \mathcal{C} \rightarrow \pi_{0} \mathcal{D}$ is an equivalence of categories, proving condition W2.

It remains to show that $f$ satisfies property F2. By Proposition 2.3 and the fact that satisfying $\mathrm{F} 1$ is equivalent to having the right lifting property with respect to maps in A1, it suffices to show that $f$ has the right lifting property with respect to the maps in A2. But, a map $\{x\} \rightarrow \mathcal{H}$ in A2 can be written as a (possibly infinite) composition of a pushout along $\phi \rightarrow\{x\}$ followed by pushouts along maps of the form $U \dot{\Delta}[n] \rightarrow U \Delta[n]$, and $f$ has the right lifting property with respect to all such maps since these are just the maps in $\mathrm{C} 1$ and $\mathrm{C} 2$.

Proposition 3.3. A map in SC is an acyclic cofibration if and only if it has the left lifting property with respect to the fibrations.

The proof will require the use of the following lemma:

Lemma 3.4. Let $\mathcal{A} \rightarrow \mathcal{B}$ be a map in $A 1$ or $A 2$ and $i: \mathcal{A} \rightarrow \mathcal{C}$ any map in SC. Then in the pushout diagram

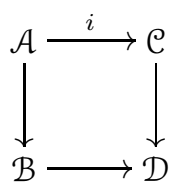

the map $\mathcal{C} \rightarrow \mathcal{D}$ is a weak equivalence.

Proof. First suppose that the map $\mathcal{A} \rightarrow \mathcal{B}$ is in A2. Let $\mathcal{O}$ be the set of objects of $\mathcal{C}$ and define $\mathcal{O}^{\prime}$ to be the set $\mathcal{O} \backslash\{x\}$. (For simplicity of notation, we assume that $i x=x$.) Assume as before that $x$ and $y$ are the objects of $\mathcal{H}$. We denote also by $\mathcal{O}^{\prime}$ the (simplicial) category with object set $\mathcal{O}^{\prime}$ and no nonidentity morphisms. Consider the diagram

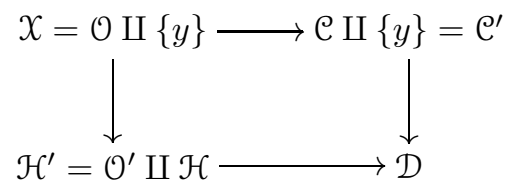

and notice that $\mathcal{D}$ is also the pushout of this diagram. Since $\mathcal{X}$ (regarded as a set) is the object set of any of these categories, note that the left-hand vertical arrow is a cofibration in $\mathcal{S} \mathcal{C}_{x}$.

We factor the map $X \rightarrow \mathcal{C}^{\prime}$ as the composite of a cofibration and an acyclic fibration in $\mathcal{S C}_{x}$

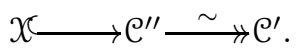


Since $\mathcal{S C}_{x}$ is proper [4, 7.3], it follows from [7, 13.5.4] that the pushouts of each row in the diagram

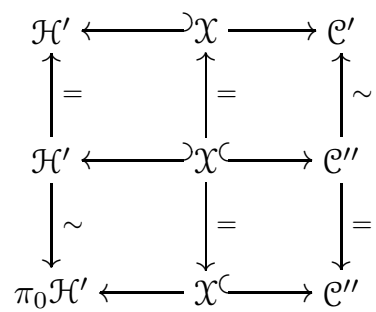

are weakly equivalent to one another. In particular, the pushout of the bottom row is weakly equivalent to $\mathcal{D}$. It remains to show that there is a weak equivalence of pushouts of the rows of the diagram

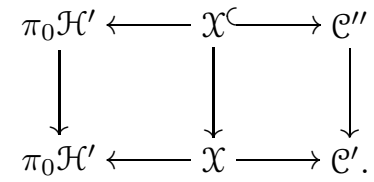

However, a calculation shows that the pushout of this bottom row is weakly equivalent in $\mathcal{S} \mathcal{C}$ to the pushout of the diagram

$$
\pi_{0} \mathcal{H} \longleftarrow\{x\} \longrightarrow \mathcal{C}
$$

and therefore that the pushout of the top row is weakly equivalent to the pushout of the bottom row. It follows that the map $\mathcal{C} \rightarrow \mathcal{D}$ is a weak equivalence in $\mathcal{S}$.

For the maps in A1, we have pushout diagrams

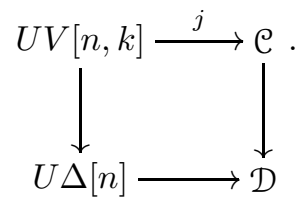

As before, define $\mathcal{O}$ to be the object set of $\mathcal{C}$. Let $\mathcal{O}^{\prime \prime}=\mathcal{O} \backslash\{x, y\}$. (Again, for notational simplicity we will assume that $j x=x$ and $j y=y$.) Now we consider the diagram

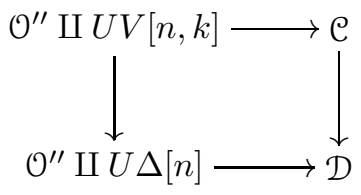

in $\mathcal{S C}_{\mathcal{O}}$. However, since the left vertical map is a weak equivalence and assuming that the top map is a cofibration (factoring if necessary as above), we can again use the fact that $\mathcal{S C}_{\mathcal{O}}$ is proper to show that $\mathcal{C} \rightarrow \mathcal{D}$ is a weak equivalence in $\mathcal{S C}_{\mathcal{O}}$ and thus also in $\mathcal{S} \mathcal{C}$.

Proof of Proposition 3.3. First suppose that a map $\mathcal{C} \rightarrow \mathcal{D}$ is an acyclic cofibration. By the small object argument ([5, Sec. 7] or [7, Ch. 11]), we have a factorization of the map $\mathcal{C} \rightarrow \mathcal{D}$ as the composite $\mathcal{C} \rightarrow \mathcal{C}^{\prime} \rightarrow \mathcal{D}$ where $\mathcal{C}^{\prime}$ is obtained from $\mathcal{C}$ by a directed colimit of iterated pushouts along the maps in A1 and A2. Thus, by Lemma 3.4 above and the fact that a directed colimit of such maps is a weak equivalence, this map $\mathcal{C} \rightarrow \mathcal{C}^{\prime}$ is a weak equivalence. Furthermore, the map $\mathcal{C}^{\prime} \rightarrow \mathcal{D}$ 
has the right lifting property with respect to the maps in A1 and A2. Thus, by Proposition 2.3, it is a fibration. It is also a weak equivalence since the maps $\mathcal{C} \rightarrow \mathcal{D}$ and $\mathcal{C} \rightarrow \mathcal{C}^{\prime}$ are, by model category axiom MC2. In particular, by the definition of cofibration, it has the right lifting property with respect to the cofibrations. Therefore, there exists a dotted arrow lift in the diagram

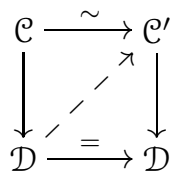

Hence the map $\mathcal{C} \rightarrow \mathcal{D}$ is a retract of the map $\mathcal{C} \rightarrow \mathcal{C}^{\prime}$ and therefore also has the left lifting property with respect to fibrations.

Conversely, suppose that the map $\mathcal{C} \rightarrow \mathcal{D}$ has the left lifting property with respect to fibrations. In particular, it has the left lifting property with respect to the acyclic fibrations, so it is a cofibration by definition. We again obtain a factorization of this map as the composite $\mathcal{C} \rightarrow \mathcal{C}^{\prime} \rightarrow \mathcal{D}$ where $\mathcal{C}^{\prime}$ is obtained from $\mathcal{C}$ by iterated pushouts of the maps in $\mathrm{A} 1$ and A2. Once again, the map $\mathcal{C}^{\prime} \rightarrow \mathcal{D}$ has the right lifting property with respect to the maps in A1 and A2 and thus is a fibration by Proposition 2.3. Therefore there is a lift in the diagram

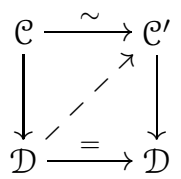

Again using Lemma 3.4 the map $\mathcal{C} \rightarrow \mathcal{D}$ is a weak equivalence because it is a retract of the map $\mathcal{C} \rightarrow \mathcal{C}^{\prime}$.

We have now proved everything we need for the existence of the model category structure on SC.

Proof of Theorem 1.1. It remains to show that the four conditions of Proposition 1.2 are satisfied. It can be shown that both $\phi$ and $\{x\}$ are small, and using the smallness of $V[n, k]$ and $\Delta[n]$ in SSets [8, 3.1.1], it can be shown that each $U \dot{\Delta}[n]$ is small relative to the set $\mathrm{C} 1$ and each $U V[n, k]$ is small relative to the set A1 [7, 10.5.12]. Therefore, condition 1 holds. Condition 2 follows from Propositions 2.3 and 2.5. Condition 3 is proved in Proposition [3.2, and condition 4 is proved in Proposition 3.3 .

We conclude this section with the following result about this model category structure.

Proposition 3.5. The model category structure SC is right proper.

Proof. Suppose that

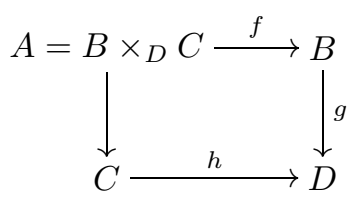

is a pullback diagram, where $g: B \rightarrow D$ is a fibration and $h: C \rightarrow D$ is a DK-equivalence. We would like to show that $f: A \rightarrow B$ is a DK-equivalence. 
We first need to show that $\operatorname{Hom}_{A}(x, y) \rightarrow \operatorname{Hom}_{B}(f x, f y)$ is a weak equivalence of simplicial sets for any objects $x$ and $y$ of $A$. However, this fact follows since the model category structure on simplicial sets is right proper [7, 13.1.4].

It remains to prove that $\pi_{0} A \rightarrow \pi_{0} B$ is an equivalence of categories. After applying $\pi_{0}$ to the result of the previous paragraph, it suffices to show that $A \rightarrow B$ is essentially surjective on objects.

Consider an object $b$ of $B$ and its image $g(b)$ in $D$. Since $C \rightarrow D$ is a DKequivalence, there exists some object $c$ of $C$ together with a homotopy equivalence $g(b) \rightarrow h(c)$ in $D$. Since $B \rightarrow D$ is a fibration, there exists an object $b^{\prime}$ and homotopy equivalence $b \rightarrow b^{\prime}$ in $B$ such that $g\left(b^{\prime}\right)=h(c)$. Using the fact that $A$ is a pullback, we have a homotopy equivalence $b \rightarrow f\left(\left(b^{\prime}, c\right)\right)$, completing the proof.

\section{Proof of Lemma 2.4}

Recall that we have a (simplicial) category $\mathcal{F}$ with objects $x$ and $y$ and a single nonidentity morphism $g: x \rightarrow y$, and a simplicial category $\mathcal{E}^{\prime}$ also with objects $x$ and $y$ such that there is a map $i: \mathcal{F} \rightarrow \mathcal{E}^{\prime}$ which sends $g$ to a homotopy equivalence $x \rightarrow y$ in $\mathcal{E}^{\prime}$. We first replace $\mathcal{E}^{\prime}$ by its subcategory of homotopy equivalences which we denote by $\mathcal{E}$. In order to make our constructions homotopy invariant, we take functorial cofibrant replacements $\widetilde{\mathcal{F}} \rightarrow \mathcal{F}$ and $\widetilde{\mathcal{E}} \rightarrow \mathcal{E}$ in the model category $\mathcal{S \mathcal { C }}_{\{x, y\}}$ as given in [4, 2.5], and in this construction $\widetilde{\mathcal{F}}$ is actually isomorphic to $\mathcal{F}$.

Now, take the localization $\mathcal{F}^{-1} \mathcal{F}$ (respectively $\widetilde{\mathcal{E}}^{-1} \widetilde{\mathcal{E}}$ ) obtained by formally inverting all the morphisms in each simplicial degree of $\mathcal{F}$ (respectively $\widetilde{\mathcal{E}}$ ). These localizations are the groupoid completions of $\mathcal{F}$ and $\widetilde{\mathcal{E}}$, respectively. (In taking a functorial cofibrant replacement and then the groupoid completion, we have taken the simplicial localizations of $\mathcal{F}$ and $\mathcal{E}$ with respect to all the morphisms in each as defined in [4.) We now have a diagram

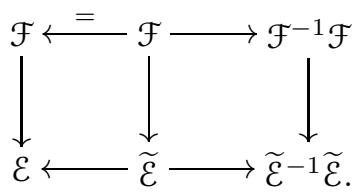

To assure that our next step is homotopy invariant, we factor the map $\mathcal{F}^{-1} \mathcal{F} \rightarrow$ $\widetilde{\mathcal{E}}^{-1} \widetilde{\mathcal{E}}$ as the composite

$$
\mathcal{F}^{-1} \mathcal{F} \stackrel{i}{\longrightarrow} Z \stackrel{p}{\longrightarrow} \widetilde{\mathcal{E}}^{-1} \widetilde{\mathcal{E}}
$$

where $i$ is an acyclic cofibration and $p$ is a fibration in $\mathcal{S C}_{\{x, y\}}$. However, to avoid more notation than necessary, we will assume that the map $\mathcal{F}^{-1} \mathcal{F} \rightarrow \widetilde{\mathcal{E}}^{-1} \widetilde{\mathcal{E}}$ is a fibration and continue to write $\mathcal{F}^{-1} \mathcal{F}$ rather than $\mathcal{Z}$.

We take the pullback of the bottom right-hand corner of the above diagram and denote it 9 :

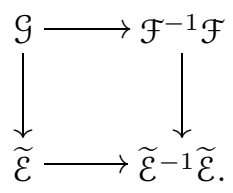

Notice that this diagram is a pullback in $\mathcal{S C}_{\{x, y\}}$ as well as in $\mathcal{S} \mathcal{C}$. 
Lemma 4.1. The composite map $\{x\} \rightarrow \mathcal{F} \rightarrow \mathcal{G}$ is a weak equivalence in $\mathcal{S} \mathcal{C}$.

Proof. Since the simplicial categories $\widetilde{\mathcal{E}}, \widetilde{\mathcal{E}}-1 \widetilde{\mathcal{E}}$, and $\mathcal{F}^{-1} \mathcal{F}$ all consist of homotopy equivalences, so must $\mathcal{G}$. Therefore, all the morphisms of $\pi_{0} \mathcal{G}$ are isomorphisms, and in particular, the objects $x$ and $y$ are isomorphic in $\pi_{0} \mathcal{G}$.

It then suffices to show that $\mathcal{G}$ has weakly contractible function complexes. Because all of the morphisms of $\mathcal{E}$, and hence also of $\widetilde{\mathcal{E}}$, are homotopy equivalences, the map $\widetilde{\mathcal{E}} \rightarrow \widetilde{\mathcal{E}}^{-1} \widetilde{\mathcal{E}}$ is a weak equivalence in $\mathcal{S C}_{\{x, y\}}$ [4, 9.5].

Note that $\mathcal{F}^{-1} \mathcal{F}$ is the simplicial category in $\mathcal{S C}_{\{x, y\}}$ with exactly one morphism between any two objects. In particular, $\mathcal{F}^{-1} \mathcal{F}$ has weakly contractible function complexes.

Now, because all the categories have as objects $x$ and $y$ and all the maps involved are the identity on these objects, we can consider the above pullback diagram in $\mathcal{S C}_{\{x, y\}}$. Since this model category structure is right proper [4, 7.3], every pullback of a weak equivalence along a fibration is a weak equivalence. The map $\widetilde{\mathcal{E}} \rightarrow \widetilde{\mathcal{E}}^{-1} \widetilde{\mathcal{E}}$ is a weak equivalence and the map $\mathcal{F}^{-1} \mathcal{F} \rightarrow \widetilde{\mathcal{E}}^{-1} \widetilde{\mathcal{E}}$ is a fibration, so it follows that the map $\mathcal{G} \rightarrow \mathcal{F}^{-1} \mathcal{F}$ is a weak equivalence in $\mathcal{S C}_{\{x, y\}}$, and therefore $\mathcal{G}$ has weakly contractible function complexes. Thus, the map $\{x\} \rightarrow \mathcal{G}$ satisfies the conditions to be a weak equivalence in $\mathcal{S} \mathcal{C}$.

However, not all the maps $\{x\} \rightarrow \mathcal{G}$ are isomorphic to maps in A2 because the simplicial categories $\mathcal{G}$ could have an uncountable number of simplices in their function complexes. Furthermore, there is no reason to assume that the inclusion map $\{x\} \amalg\{y\} \rightarrow \mathcal{G}$ is a cofibration in $\mathcal{S}_{\{x, y\}}$. To complete the proof, we need to show that any acyclic cofibration $\{x\} \rightarrow \mathcal{G}$ as above factors as a composite $\{x\} \rightarrow \mathcal{H} \rightarrow \mathcal{G}$ where the inclusion map $\{x\} \rightarrow \mathcal{H}$ is in A2.

Let $\mathcal{H}_{0}$ be the simplicial category $\mathcal{F}$. Let $i: \mathcal{H}_{0} \rightarrow \mathcal{G}$ be the inclusion map. We will construct a simplicial category $\mathcal{H}$ from $\mathcal{H}_{0}$ satisfying the necessary properties specified in A2. We first state the following lemma:

Lemma 4.2. Let $f: A \rightarrow B$ be a map of simplicial sets where $B$ is weakly contractible, and let $u: S^{n} \rightarrow|A|$ be a map of $C W$-complexes for some $n \geq 0$. Then $f$ can be factored as a composite $A \rightarrow A^{\prime} \rightarrow B$ where $A^{\prime}$ is obtained from $A$ by attaching a finite number of nondegenerate simplices and the composite map of spaces $S^{n} \rightarrow|A| \rightarrow\left|A^{\prime}\right|$ is null homotopic.

Proof. We first assume that the map $f$ is a cofibration; if not, we factor it as the composite

$$
A \stackrel{i}{\longrightarrow} B^{\prime} \stackrel{p}{\longrightarrow} B
$$

where in the model category structure on simplicial sets $i$ is a cofibration and $p$ is an acyclic fibration. Thus, we can assume that $f$ is an inclusion map, replacing $B$ by $B^{\prime}$ if needed.

Now consider the composite map of spaces $S^{n} \rightarrow|A| \rightarrow|B|$, which is necessarily null homotopic since $B$ is weakly contractible. The composite map then factors through $C S^{n}$, the cone on $S^{n}$, and we have a diagram

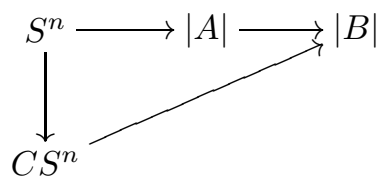


Now, since $C S^{n}$ is compact, its image will intersect only a finite number of cells of $|B|$ nontrivially. Then define $A^{\prime}$ to be a simplicial set such that $\left|A^{\prime}\right|$ contains $|A|$ as well as all the cells in this image.

Now, consider the categories $\mathcal{H}_{0}$ and $\mathcal{G}$ as described above and the inclusion map $i: \mathcal{H}_{0} \rightarrow \mathcal{G}$. Each of these categories has four function complexes to consider. For the category $\mathcal{H}_{0}$ we call them $H_{j}$, and for $\mathcal{G}$ we call them $G_{j}$ for $1 \leq j \leq 4$. (The numbering is arbitrary but must match up between the two categories. So if $H_{1}=\operatorname{Hom}_{\mathcal{H}}(x, y)$, then we must have $G_{1}=\operatorname{Hom}_{\mathcal{G}}(x, y)$.)

First notice that $\operatorname{Hom}_{\mathcal{H}_{0}}(y, x)=\phi$. We begin by adding a map $y \rightarrow x$ to make this function complex nonempty, as well as all composites generated by it. Then, considering all four function complexes, we identify $n \geq 0$ such that all maps $S^{m} \rightarrow\left|H_{j}\right|$ are null homotopic for all $0 \leq m<n$ and all $1 \leq j \leq 4$, but there is a map $S^{n} \rightarrow\left|H_{j}\right|$ which is not null homotopic for some $j$. We then apply Lemma 4.2 to the map $H_{j} \rightarrow G_{j}$ and the map $S^{n} \rightarrow\left|H_{j}\right|$.

Replace the function complex $H_{j}$ with the simplicial set $A^{\prime}$ obtained from Lemma 4.2. This process may result in more maps $S^{m} \rightarrow\left|A^{\prime}\right|$ which are not null homotopic than for the original $\left|H_{j}\right|$, but only for $m>n$. Also, it will not have more than countably many more such maps than $\left|H_{j}\right|$ did. Now that we have added simplices to our function complex, we include all necessary compositions of these morphisms with the original morphisms of $\mathcal{H}_{0}$ to obtain a new simplicial category which we denote $\mathcal{H}_{1}$. There will be at most countably many new simplices added from these compositions. Repeat the above process with another map from $S^{n}$ to a function complex of $\mathcal{H}_{1}$, again, where $n$ is minimal, to obtain another category $\mathcal{H}_{2}$. Continue, perhaps countably many times, to obtain a category $\mathcal{H}$ such that for any $n$ and any function complex $H^{\prime}$ of $\mathcal{H}$, any map $S^{n} \rightarrow\left|H^{\prime}\right|$ is nullhomotopic. To show that it is possible to obtain such an $\mathcal{H}$ in this way, we need only show that there are at most countably many homotopy classes of maps from spheres to each function complex that need to be killed off. However, this fact follows from the following lemma:

Lemma 4.3. Let $A$ be a simplicial set with countably many simplices. Then for all $n \geq 0$ there are at most countably many distinct homotopy classes of maps $S^{n} \rightarrow|A|$.

Proof. It suffices to show that there are at most countably many homotopy classes of maps from $S^{n}$ into any finite CW complex $X$. For a simply connected CW complex $X$, an argument using Serre mod $\mathcal{C}$ theory [9] shows that all the homotopy groups of $X$ are countable if and only if the homology groups of $X$ are countable, which they are when $X$ is finite. The case of a general CW complex $X$ follows from this one using a universal cover argument.

By construction, this simplicial category $\mathcal{H}$ is free, and therefore the map

$$
\{x\} \amalg\{y\} \rightarrow \mathcal{H}
$$

is a cofibration in $\mathcal{S C}_{\{x, y\}}$. Thus, we have obtained a factorization $\{x\} \rightarrow \mathcal{H} \rightarrow \mathcal{G}$. We are now able to complete the proof of Lemma 2.4.

Proof of Lemma 2.4. Using the simplicial category $\mathcal{H}$ from above and the map $\mathcal{H} \rightarrow \mathcal{G}$, we obtain a composite map

$$
\{x\} \rightarrow \mathcal{F} \rightarrow \mathcal{H} \rightarrow \mathcal{G} \rightarrow \widetilde{\mathcal{E}} \rightarrow \mathcal{E} \rightarrow \mathcal{E}^{\prime} .
$$


In particular, we have a factorization $\mathcal{F} \rightarrow \mathcal{H} \rightarrow \mathcal{E}^{\prime}$. As we have shown above, the composite $\{x\} \rightarrow \mathcal{F} \rightarrow \mathcal{H}$ is isomorphic to a map in A2.

\section{ACKNOWLEDGMENT}

The author would like to thank Bill Dwyer for helpful conversations about this paper.

\section{REFERENCES}

[1] J.E. Bergner, Three models for the homotopy theory of homotopy theories, Ph.D. Thesis, University of Notre Dame, 2005.

[2] William G. Dwyer, Philip S. Hirschhorn, Daniel M. Kan, and Jeffrey H. Smith, Homotopy limit functors on model categories and homotopical categories. Mathematical Surveys and Monographs, 113. American Mathematical Society, Providence, RI, 2004. MR2102294 (2005k:18027)

[3] W.G. Dwyer and D.M. Kan, Function complexes in homotopical algebra, Topology 19 (1980), 427-440. MR0584566 (81m:55018)

[4] W.G. Dwyer and D.M. Kan, Simplicial localizations of categories, J. Pure Appl. Algebra 17 (1980) 267-284. MR0579087 (81h:55018)

[5] W.G. Dwyer and J. Spalinski, Homotopy theories and model categories, in Handbook of Algebraic Topology, Elsevier, 1995. MR1361887 (96h:55014)

[6] P.G. Goerss and J.F. Jardine. Simplicial Homotopy Theory, Progress in Math, vol. 174, Birkhauser, 1999. MR.1711612 (2001d:55012)

[7] Philip S. Hirschhorn, Model Categories and Their Localizations, Mathematical Surveys and Monographs 99, AMS, 2003. MR1944041 (2003j:18018)

[8] Mark Hovey, Model Categories, Mathematical Surveys and Monographs 63, AMS, 1999. MR1650134 (99h:55031)

[9] Jean-Pierre Serre, Groupes d'homotopie et classes de groupes abéliens, Ann. of Math, 58 (1953), 258-294. MR0059548 (15:548c)

[10] Bertrand Toën and Gabriele Vezzosi. Homotopical algebraic geometry I: topos theory, preprint available at math.AG/0207028.

Department of Mathematics, University of Notre Dame, Notre Dame, Indiana 46556

E-mail address: bergnerj@member.ams.org

Current address: Department of Mathematics, Kansas State University, 138 Cardwell Hall, Manhattan, Kansas 66506 